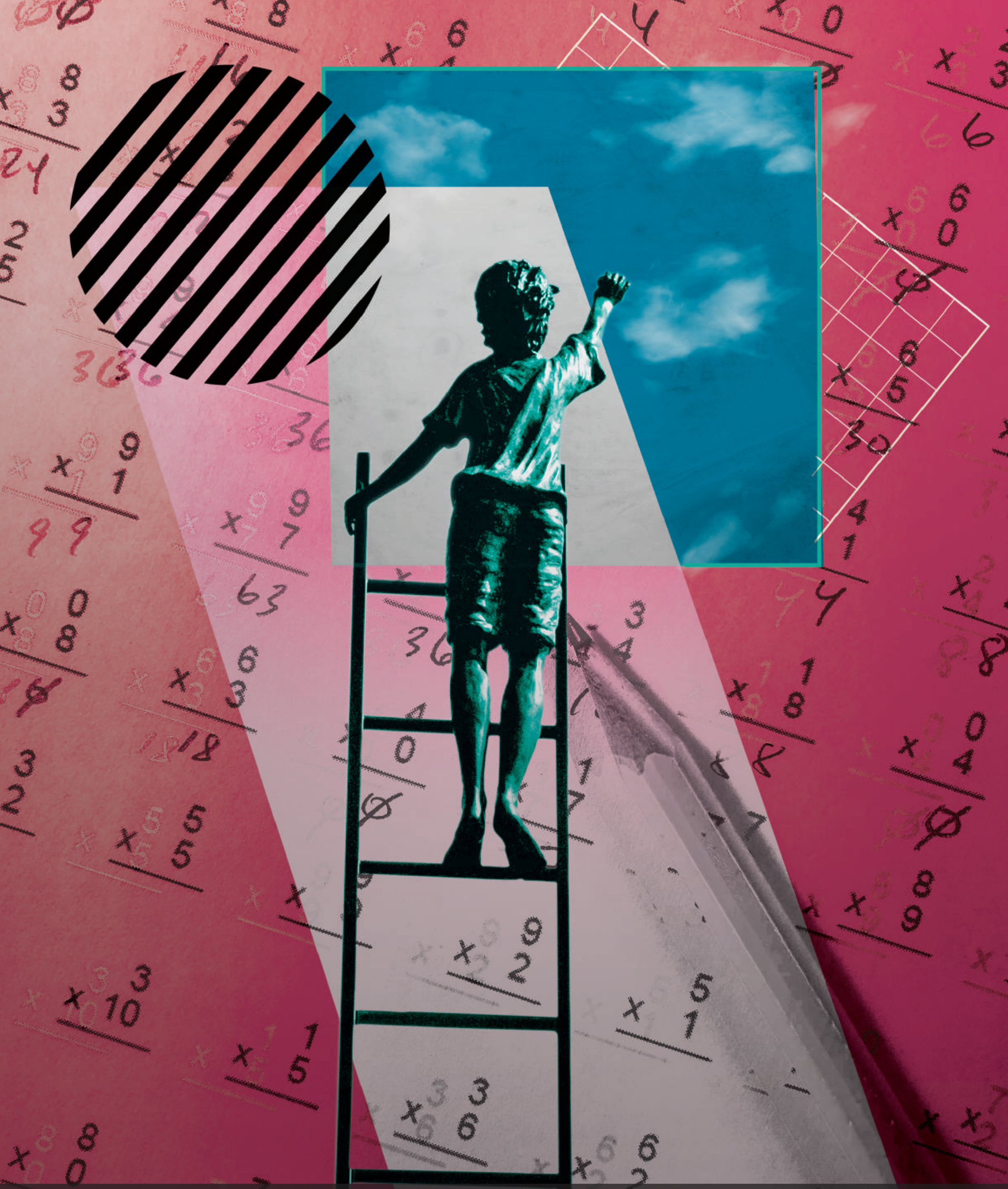




\section{FORMACIÓN DOCENTE Y DESARROLLO PROFESIONAL SITUADO PARA LA ENSEÑANZA DEL LENGUAJE Y MATEMÁTICAS EN COLOMBIA

\section{Teacher Training And Professional Development Located For'The Teaching Of Language And Mathematics In Colombia}

Formação de professores e desenvolvimento profissional localizados para o ensino da linguagem e matemática na Colômbia

\section{RECIBIDO:3 MARZO 2017}

Alfredo González (Colombia)

Universidad Nacional de Rosario-Argentina

Candidato a Doctor en Educación alfredmat911@gmail.com

EVALUADO:10MAYO 2017,

16 AGOSTO 2017

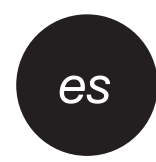

\section{RESUMEN}

Este artículo tiene como propósito presentar un rastreo bibliográfico de las tendencias actuales en Colombia acerca de la formación docente y desarrollo profesional situado para la enseñanza del Lenguaje y Matemáticas en los niveles de educación básica primaria y media académica. Se pretende focalizar en tres categorías de análisis: comunidades de aprendizaje, desarrollo profesional situado y práctica pedagógica, demostrando de manera concreta cómo se ha impactado en la calidad de la educación del país, desde el sector oficial, mediante el análisis de los resultados obtenidos en las investigaciones realizadas en este campo educativo. Estas categorías desarrollan algunas dimensiones que permiten resolver el interrogante de cuáles son los cambios que se dan en la práctica pedagógica de los docentes luego de aplicar un entrenamiento en la metodología del estudio de clases. El análisis de este sistema de categorías y dimensiones se ha realizado bajo el proceso de la enseñanza del Lenguaje y Matemáticas, y de cómo este contribuye significativamente en el mejoramiento de los procesos de comprensión de lectura y resolución de problemas matemáticos. Los estudios abordados demuestran un efecto positivo sobre la enseñanza e instrucción orientada por los docentes, especialmente en sus metodologías, estrategias didácticas, desarrollo profesional situado y las prácticas evaluativas, así como en el aprendizaje de los estudiantes. Esto se materializa en la consolidación de comunidades de aprendizaje conformadas por los docentes de lenguaje y matemáticas del país -teniendo en cuenta áreas de conocimiento y perfil de cada uno de ellos-, donde se realizan constantemente procesos de planeación, ejecución, observación y retroalimentación de las clases, por medio de equipos de estudio

PALABRAS CLAVE: comunidad de aprendizaje, calidad educativa, evaluación, formación docente, desarrollo profesional situado.

\section{en}

ABSTRACT

The purpose of this article is to present a bibliographic survey of the current trends

in Colombia regarding teacher training and professional development located for the teaching of Language and Mathematics at primary and secondary academic education levels. The aim is to focus on three categories of analysis: learning communities, situated professional development and pedagogical practice, demonstrating in a concrete way how the quality of education in the country has been impacted, from the official sector, by analyzing the results obtained in the research carried out in this educational field. These categories develop some dimensions that allow to solve the question of what are the changes that occur in the pedagogical practice of the teachers after applying a training in the methodology of the study of classes. The analysis of this system of categories and dimensions has been carried out under the process of teaching Language and Mathematics, and how it contributes significantly in improving the processes of reading comprehension and solving mathematical problems. The studies addressed demonstrate a positive effect on teaching and instruction oriented by teachers, especially in their methodologies, didactic strategies, situated professional development and evaluative practices, as well as in student learning.

KEYWORDS: Community of learning, educational quality, evaluation, teacher training, professional development located.

\section{ACEPTADO:22 NOVIEMBRE 2017}

Alex Mauricio Díaz (Colombia) alexdiazd@correo.unicordoba.edu.co Universidad Nacional de Rosario-Argentina Candidato a Doctor en Educación

\section{por}

\section{RESUMO}

O objetivo deste artigo é apresentar um levantamento bibliográfico sobre as tendências atuais da Colômbia no que se refere à formação de professores e desenvolvimento profissional localizado para o ensino de Língua e Matemática nos níveis de ensino acadêmico primário e secundário. Destina-se a concentrarse em três categorias de análise: comunidades de aprendizagem, situado desenvolvimento profissional e prática de ensino, demonstrando concretamente como isso tem impactado a qualidade da educação no país, o setor oficial, através da análise dos resultados a pesquisa realizada nesse campo educacional. Essas categorias desenvolvem algumas dimensões que permitem resolver a questão de quais são as mudanças que ocorrem na prática pedagógica dos profesores após a aplicação de um treinamento na metodologia do estudo das aulas. A análise deste sistema de categorias e dimensões tem sido realizada no processo de ensino de Linguagem e Matemática, e como contribui significativamente na melhoria dos processos de compreensão de leitura e resolução de problemas matemáticos. Os estudos abordados demonstram um efeito positivo no ensino e na instrução orientados pelos professores, especialmente em suas metodologias, estratégias didáticas, desenvolvimento profissional situado e práticas avaliativas, bem como na aprendizagem dos alunos.

PALAVRAS CHAVE: Comunidade de aprendizagem, qualidade educacional, avaliação, formação de professores, desenvolvimento profissional localizado. 


\section{INTRODUCCIÓN}

En Colombia, en los últimos años, las políticas públicas de educación se han orientado a resolver el problema de cobertura, permanencia y repitencia a partir de múltiples acciones institucionales y gubernamentales, aislando el tema de la calidad educativa, lo cual se ve reflejado en los bajos niveles de desempeño de los estudiantes del país en las áreas de lenguaje y matemáticas. De acuerdo con los resultados para las pruebas Saber $3^{\circ}, 5^{\circ}$ y $9^{\circ}$ que se presentaron en el año 2014, hay un alto porcentaje de los estudiantes que presentan niveles de desempeños bajos e insuficientes en lenguaje y matemáticas (Icfes, 2018). Por esta razón, una de las metas del gobierno colombiano actual es la de alcanzar para el año 2025 la calidad educativa ${ }^{1}$, sin embargo, esta meta solo puede ser concretada con prácticas de aula pertinentes, eficaces y significativas. El sistema educativo debe garantizar aprendizajes básicos, universalmente definidos como lo son el aprendizaje efectivo del lenguaje y las competencias matemáticas, las cuales son el sustento esencial para los demás aprendizajes dado que estas dos áreas se constituyen en los pilares de la educación básica primaria, secundaria, media y superior.

En consonancia con lo anterior, la sociedad en la que nos desenvolvemos hoy en día exige una educación en donde nuestros jóvenes sean ciudadanos competentes en un medio cada vez más exigente que evoluciona con criterios de calidad y demanda mayor capacidad de adaptación a las nuevas circunstancias. Esto implica usar el lenguaje como medio que nos permita crear, procesar variadas informaciones y establecer múltiples interacciones. Se hace necesario, entonces, incentivar en nuestros estudiantes el desarrollo de habilidades y competencias para la adquisición del pensamiento matemático y el funcionamiento del lenguaje, de tal forma que se pueda propiciar la creación de significados y se pueda cumplir con el propósito comunicativo y competencias científicas del proceso educativo. En este sentido, en los últimos años muchos sistemas educativos han puesto especial atención a la medición de los resultados del aprendizaje en un intento por evaluar la calidad de sus

1 En el año 2015, en Colombia se expidió la Ley 1753, denominada Ley del Plan Nacional de Desarrollo 2014-2018 “Todos por un nuevo país”. A partir de esta norma, se desarrolló un plan ambicioso para materializar el propósito de hacer de Colombia el país mejor educado de América Latina en el año 2025. En ese marco, se plantearon las líneas estratégicas que determinarán el rumbo de la educación para los próximos años: Excelencia Docente, Jornada Única, Colombia Bilingüe, Colombia Libre de Analfabetismo y Más 8 Acceso a la Educación Superior de Calidad. MEN (2015). sistemas de educación y por emplear los resultados obtenidos de esas mediciones para orientar las decisiones en materia de políticas educativas (UNESCO, 2014).

Luego entonces, las prácticas de aula se constituyen en una de las herramientas claves que contribuyen con la consecución del objetivo de la calidad educativa planteada por el Estado. Una de las estrategias que se proponen para mejorar las prácticas de aula y el proceso de enseñanza de los docentes es la implementación del modelo de desarrollo de formación docente. Este, por consiguiente, necesita dirigirse al colectivo de maestros y debe ser con formación situada, esto quiere decir que debe desarrollarse en el contexto y en relación con la práctica de aula y sus problemáticas específicas. A partir de esto, es necesario reconsiderar el trabajo docente y su acción consecuente en las aulas, en el sentido que la formación docente debe examinar conceptualmente la naturaleza de la disciplina y sus formas apropiadas de representación y de enseñanza para los distintos tipos de estudiantes (Shulman, 1987, citado por Avalos, 2006). Entonces, el desarrollo profesional docente debe verse como un proceso que implica aprender y transformar la propia práctica de manera continua, con el fin de potenciar la capacidad para enfrentar los nuevos retos, para descubrir los que van surgiendo y sobre los que hay que aprender otra vez.

Lo anterior significa una educación que asegure mínimamente, en los niños y jóvenes, la adquisición de los conocimientos, capacidades, destrezas y actitudes necesarias para desempeñarse en la vida adulta. De esta manera, el mejoramiento de la calidad y el aprendizaje ha de tener un papel más decisivo en el marco del desarrollo mundial en los próximos años. Por eso, el diseño de estrategias es un factor clave, si se busca mejorar las posibilidades de educación de millones de niños que son incapaces de leer y escribir o no disponen de competencias aritméticas básicas ${ }^{2}$. En tal sentido, se presenta una crisis del aprendizaje en nuestros estudiantes, de la que resulta necesario ocuparse, si se quiere formar en competencias en áreas como el lenguaje y matemáticas, puesto que son necesarias para conseguir un trabajo

La UNESCO (2014), publicó el undécimo Informe de seguimiento de la Educación Para Todos (EPT). Aquí se muestran los avances logrados por los diferentes países en la consecución de los objetivos mundiales de educación acordados en el año 2000. También se presentan argumentos convincentes en favor de que la educación ocupe un lugar central en la agenda mundial para el desarrollo después de 2015. 
digno que les permita la realización y el desarrollo a plenitud de su proyecto de vida. Por esta razón, es necesario contar con docentes capacitados, con dedicación suficiente, que sepan descubrir y prestar atención especial a quienes tienen dificultades de aprendizaje, y que estén apoyados, a su vez, por sistemas educativos bien administrados, de modo que puedan liberar su potencial y contribuir al mejoramiento de la calidad de los aprendizajes de sus estudiantes.

Ahora bien, existe un vacío para lograr la formación de excelentes profesionales de la educación, puesto que debe existir un proceso de profesionalización de la labor docente que apunte a que se tenga mayor conocimiento de la enseñanza a lo largo de la carrera. Países como Finlandia o Corea exhiben docentes de las más altas calidades, con procesos de selección competitivos y rigurosos y una posición social importante. Así, enseñar de forma sistemática y efectiva es una actividad profesional altamente especializada que requiere entrenamiento, como en cualquier otra profesión (Ball y Forzani, 2009). En este proceso de formación profesional docente, considerado como factor clave para la calidad de la educación, se debe considerar que, al mejorar la acción educativa, se puede conseguir al mismo tiempo mayores niveles de calidad en el funcionamiento de las instituciones educativas (Marchesi, 2011). Esto sugiere que la calidad de la educación mejora cuando se apoya a los docentes y cuando las propuestas para mejorar su situación se basan en enfoques contextuales e integrales, en los que se tengan en cuenta todos los factores que contribuyen a facilitar el trabajo de los docentes, puesto que ellos no pueden cargar con toda la responsabilidad de los problemas asociados a la calidad de la educación. Sin embargo, hoy la Educación de Calidad en Colombia ha sido definida como aquella que permite formar mejores seres humanos, ciudadanos con valores éticos, respetuosos de lo público, que ejercen los derechos humanos y conviven en paz. Se propone una educación que genere oportunidades legítimas de progreso y prosperidad, que sea competitiva, y que contribuya a cerrar brechas de inequidad (MEN, 2011).

A continuación se presenta una revisión bibliográfica y rastreo de antecedentes acerca de la importancia de las comunidades de aprendizaje de docentes ${ }^{3}$ en los sistemas educativos. Estas se definen, de acuerdo con

\footnotetext{
3 En lo sucesivo del texto se utilizará la abreviatura CDA para hacer referencia a
} Comunidades de Aprendizaje de docentes.
Poehner, como: "Incitar a los maestros a hacerse cargo de sus propios aprendizajes al reflexionar y cuestionar constantemente en equipo sus prácticas en el aula, convirtiéndose en profesores investigadores, en lugar de simplemente impartidores de conocimiento" (2011). Lo que quiere decir que los docentes integrantes de una CDA se convierten en observadores, interrogadores y aprendices, formándose maestros más completos y con nociones de pares académicos, según Sergiovanni y Starratt (1998, p. 259). Este tipo de entrenamiento genera en los docentes una competencia de trabajo en equipo, fortaleciendo en este sentido el aprendizaje colaborativo y cooperativo desde su praxis educativa, lo cual permite ganar experiencia desde ambos roles: docente e investigador. También Allen y Blythe (2004), argumentan que la investigación colaborativa requiere que los maestros se reúnan en CDA para hablar sobre temas individuales de enseñanza y aprendizaje que surgen de las experiencias únicas de cada miembro del equipo, lo que permite estar en constante búsqueda de solución al problema educativo del contexto donde se desarrolle este tipo de entrenamiento.

Por lo anterior, Sergiovanni y Starratt (1998, citado en Beachum and Dentith, 2004) descubrieron que es más probable que los docentes busquen ayuda y consejo de otros maestros que de otras fuentes para desarrollar y mejorar sus prácticas en el aula. Además, los profesores en CDA tienden a buscar procesos sistemáticos para involucrar a sus compañeros en la resolución de problemas que abordan su práctica (Keenan, 1974), ya sea estudiando el desarrollo de los estudiantes, analizando planes de lecciones desde múltiples perspectivas, compartiendo prácticas de gestión o investigando e implementando iniciativas curriculares en el aula. En adición, Glatthorn (1987) establece que también se logra el aprendizaje del profesor mediante la supervisión de sus pares, lo que ocurre mediante la interacción en la actividad social y cognitiva de la enseñanza más que por medio de la transmisión del conocimiento. Igualmente, este autor señaló que puede abarcar el diálogo profesional, la supervisión entre iguales, el entrenamiento entre pares y el desarrollo curricular.

En el contexto colombiano, el estudio sobre CDA se ha desarrollado desde un ámbito institucional a través del Ministerio de Educación Nacional (MEN). Para el MEN (2012), los aprendizajes efectivos de los docentes suceden en su propia práctica en el aula, por lo que las
Formación docente y desarrollo profesional situado para la enseñanza del lenguaje y matemáticas en Colombia.

I Panorama

I pp. 7-17

I Volumen 12

I Número 22

I Enero-Junio

| 2018 
Alfredo González, Alex Díaz I

Panorama I

pp. 7-17

Volumen 12

Número 22

Enero-Junio

ISSN Impreso

1909-7433

E-ISSN en linea

2145-308X

. por una CDA de 35 docentes de distintas áreas y niveles de educación. Los resultados obtenidos y proyecciones se dieron a partir de que los participantes entraron

estrategias de formación situada se dan en torno a las prácticas de aula o en talleres a los cuales los docentes llevan sus problemáticas de aula para trabajarlas en CDA. En consecuencia, asumir una estructura de formación situada requiere la creación de un ambiente de intercambio y perfeccionamiento de conocimientos, actitudes y buenas prácticas en cada colectivo de maestros para alcanzar el objetivo de formar, con apoyo técnico apropiado, comunidades de aprendizaje comprometidas con procesos de mejoramiento. A partir de lo anterior, se puede concluir que la creación de una comunidad de aprendizaje de docentes permite garantizar que sea superada la barrera del frecuente aislamiento del maestro; el trabajo entre pares se constituye en una metodología de continuo uso y aplicación con los estudiantes en el desarrollo de las clases dentro y fuera del aula escolar.

Otro estudio relacionado con CDA y que fue realizado por el MEN (2011), permite inferir que las transformaciones en el campo educativo se sostienen en el tiempo a partir de innovaciones permanentes sobre los procesos de enseñanza. En este sentido, las CDA son comunidades de conocimiento y de práctica; reflexionan y encuentran soluciones a las problemáticas específicas de aula en torno a los procesos de aprendizaje de los estudiantes; comparten inquietudes e identifican colectivamente alternativas pedagógicas. De manera que es posible distinguir algunas características de las CDA, en tanto que investigan, documentan sus experiencias, comparten sus prácticas y se nutren de las problemáticas del contexto escolar.

La experiencia 'Estrategias pedagógicas y metodológicas para la cualificación docente', proceso orientado por Cadavid (2013), cuyo propósito era implementar estrategias pedagógicas y metodológicas innovadoras que permitieran mejorar las prácticas pedagógicas de los docentes de las instituciones educativas del municipio de Manizales, permitió conocer cómo fue la implementación de las CDA en diversas instituciones. La metodología implementada durante esta experiencia educativa fue la focalización de siete instituciones educativas del municipio de Manizales del sector oficial, donde se dio el proceso de planeación, observación y evaluación en contacto real con una metodología japonesa ${ }^{4}$, pero adaptada a nuestro medio, fortaleciendo la capacitación -maestro a maestro- el trabajo colaborativo y el manejo de las TIC. Así mismo, se analizaron las ventajas de este sistema en el mejoramiento de los ambientes de aprendizaje en general, además de institucionalizar las estrategias que plantea el proyecto en las instituciones vinculadas, articulándolas a los PEI y dando continuidad a la formación de docentes en las estrategias propuestas en el proyecto investigativo.

La experiencia de implementación de Comunidad de Aprendizaje, desarrollada por Calvache y Escobar (2007), se centró en analizar las clases de los docentes, desde los aspectos de la planeación, los referentes didácticos y metodológicos empleados y la ejecución de la misma, en aras de detectar problemas en la enseñanza de la temática y sugerir nuevas estrategias, para poder así mejorar la forma de su ejecución. Con un diseño metodológico de estudios de caso, reflexión y evaluación pudieron concluir que no son pocos los beneficios aportados por el curso de mejoramiento, ya que renacen las preocupaciones y la necesidad de cambiar esas viejas prácticas pedagógicas que aún redundan en muchas instituciones del país, ya sean de carácter oficial o privado y que van desde la zona urbana hasta la rural. El curso le abrió el espacio al maestro del sector oficial para repensar su actuar docente, reflexionar en su historia como educador y vislumbrar nuevos horizontes, rutas y caminos.

Por último, este rastreo documental relacionado con CDA ha permitido establecer que hay un deseo constante de investigar acerca del impacto de esta estrategia con estudiantes de educación básica, secundaria y media, centrándose en su gran mayoría en el nivel de educación básica, tanto en otros países como en Colombia. A nivel internacional, los hallazgos aquí expresados permiten a los docentes participar en actividades de desarrollo profesional adaptadas a sus necesidades e intereses personales, proporcionando así a los docentes la posibilidad de utilizar sus aulas como un sitio para su propio desarrollo del aprendizaje. Esto, en términos de Shulman, se le conoce como el Conocimiento Didáctico de los Contenidos (CDC): la transformación de un

\footnotetext{
El 15 de octubre de 2004 se firmó entre el Ministerio de Educación Nacional de Colombia y la Agencia de Cooperación Internacional del Japón - JICA, un convenio de cooperación con el objetivo de establecer bases para el mejoramiento de la metodología de enseñanza de ciencias naturales y matemáticas en las instituciones educativas del país.
} 
buen conocimiento disciplinar en conocimiento referido a su aprendizaje requiere de razonamiento y acción.

El docente, debe interpretar los contenidos y estructurar su presentación usando un acervo de representaciones que ya posee, conformado por analogías, metáforas y ejemplos. Esto implica seleccionar las que sean apropiadas al contenido y a sus estudiantes, adaptarlas en la instrucción, evaluar su efecto, reflexionar y desarrollar a partir del proceso una nueva comprensión de este proceso pedagógico en su totalidad (Shulman, 2005).

Este desarrollo profesional crea nuevas oportunidades para reforzar los lazos colegiales que existen entre los docentes, dado que, a medida que hacen público su trabajo, buscan respuestas a problemas que no pueden resolver por sí mismos. En el contexto nacional, las investigaciones realizadas de 'Maestro invítame a tu clase. Maestros que aprenden de maestros, uso de estrategias didácticas y pedagógicas para mejorar los procesos de enseñanza', permiten hacer una reflexión acerca del desarrollo profesional docente, las comunidades de aprendizaje y la practica pedagógica -los cuales pueden coadyuvar al mejoramiento de la comprensión de lectura y los géneros textuales- se demuestra la necesidad y la pertinencia que puede tener la realización de trabajos de intervención en esta línea, así como el diseño de nuevos recursos que permitan innovar en el aula y dar solución a las problemáticas en ella, buscando generar interés hacia la lectura y, más aún, hacia el mejoramiento de la comprensión de lectura y la producción de diversos géneros textuales.

Se ha mencionado que para mejorar los aprendizajes de los estudiantes es necesario transformar las prácticas de aula, es decir, todo lo que hace el docente y sus estudiantes en el salón de clases y fuera de él. Diversas investigaciones han demostrado de forma reiterativa que lograr transformaciones en las prácticas de los docentes no es cosa fácil, y que no se resuelve solo con conferencias, talleres, discursos y/o actividades de reflexión aisladas (Díaz, 2015). En tal sentido, en este tipo de espacios, se evidencia la importancia del diálogo intelectual y profesional entre pares, que son capaces de reconocerse y reconocer sus aportes y experiencias académicas, en medio de las diferencias ideológicas, por lo que ser miembro de estos grupos de trabajo permite dar a conocer lo que sucede en nuestras aulas y volverlo objeto de conocimiento público en el marco de las comunidades de práctica y aprendizaje centradas en los aprendizajes de los estudiantes (Vescio, Ross y Adams, 2006). Es difícil cambiar la práctica docente cuando la forma como las personas enseñan se encuentra fuertemente arraigada en creencias sobre cómo se aprende y cómo se enseña. Tales creencias han sido construidas desde los primeros años de formación escolar, y desdibujan, deforman y prevalecen frente a cualquier mensaje o iniciativa de cambio que se le proponga al docente (Pajares, 1992). Consecuentemente, se requiere tomar cursos de formación in situ, acompañamiento en el aula y formación posgradual docente en niveles de maestría y doctorado (Grossman, Hammerness y McDonald, 2009), para la transformación de la calidad educativa y docente del sistema educativo actual.

Varios autores, entre ellos Furman (2013), sostienen que los cambios en las prácticas de aula requieren que los docentes se convenzan de su actitud de cambio, al ser conscientes de que sus prácticas no son efectivas. Además, que experimenten diversas formas de enseñar y aprender, dándose cuenta de lo que son capaces de hacer, y que la aplicación de dichas estrategias sea sostenible en el tiempo, de manera que puedan desarrollarlas a plenitud y consolidar aquellas que resulten como nuevas prácticas. De conseguirse esto, es posible desarrollar una estrategia de desarrollo profesional situado (DPS) ${ }^{5}$, que pueda llegar a tener un verdadero impacto en las transformaciones de las prácticas de aula. Al respecto, Abell, Rogers, Deborah y Gagnon (2009) y Putman y Borko (2000), proponen que el DPS es un pilar importante para la puesta en marcha de modelos de buenas prácticas en el aula de clase, que están representados en materiales educativos de alta calidad, sintonizados con el nivel y las posibilidades de los docentes y del medio, con el acompañamiento de tutores. Sin embargo, no se trata de simplemente llevar tutores expertos a las escuelas a realizar actividades diversas y talleres, sino más bien acompañar con ellos el desarrollo de estrategias efectivas de enseñanza-aprendizaje fundamentadas en las buenas prácticas. El DPS, requiere de diversos recursos y de un grupo de docentes bien cohesionado y motivado hacia la implantación de buenas prácticas de enseñanza en el aula.

I Panorama

I pp. 7-17

I Volumen 12

I Número 22

I Enero-Junio

I 2018

11

5 En lo sucesivo del texto se utilizará la abreviatura DPS para hacer referencia al Desarrollo Profesional Situado. 
Alfredo González, Alex Díaz

Panorama I

pp. $7-17$

Volumen 12

Número 221

Enero-Junio

2018 |

ISSN Impreso

1909-7433

E-ISSN en linea

2145-308X
En este proceso de aprendizaje entre pares, se busca desarrollar las capacidades de los miembros de un equipo de manera diferenciada para crear el saber. Los docentes se benefician porque disminuyen su aislamiento e incertidumbre y conforman verdaderas comunidades de apoyo, con el propósito de promover el desarrollo profesional y la afirmación de la identidad del maestro y su lugar como miembro valioso de la comunidad educativa (PTA, 2012). El resultado de aplicar este tipo de estrategia de planeación, observación y reflexión de la instrucción mediante el trabajo cooperativo es el mejoramiento del clima institucional y el Desarrollo Profesional Docente (DPD), lo cual es usualmente pensado como la llave para mejorar la calidad del aprendizaje de los estudiantes.

Ahora bien, en torno al proceso de cualificación es importante rescatar que los esfuerzos se han concentrado en superar las deficiencias que presentan los estudiantes en el aprendizaje de las áreas de Lenguaje y Matemáticas, especialmente en los establecimientos educativos de más bajo desempeño, según pruebas SABER. Para esto se pretende: primero, mejorar las prácticas de aula de los docentes, a través de la formación docente en posgrados y del fortalecimiento pedagógico. $\mathrm{Al}$ analizar este aspecto, es importante resaltar que los esfuerzos se enfocan a mejorar la praxis pedagógica de los docentes y sus relaciones directas con el rendimiento académico. En este punto en particular, es necesario observar que, en matemáticas y lenguaje, se han dado cambios paradigmáticos en torno a los procesos de formación de docentes, transitando de una formación no solo de tipo disciplinar, sino a un modelo que propenda porque los docentes ayuden a formar un ciudadano competente (Estándares Básicos de Competencias, 2006), donde prevalezca un modelo holístico, en palabras de Guacaneme, Obando, Garzón y Villa-Ochoa: “(...) un ciudadano competente busca trabajar sobre unas dimensiones menos pragmatistas con relación a la noción de competencia... en pos de una mirada más holística, donde el foco es la comprensión (...)” (2013).
Figura 1 Esquema de orientaciones pedagógicas y curriculares para la enseñanza del Lenguaje y Matemáticas en Colombia.

\section{REFERENTES DE CALIDAD} REFERENTES PARA LA ACTUALIZACIÓN CURRICULAR

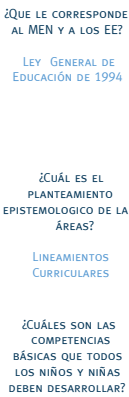

$\mathrm{EBC}$

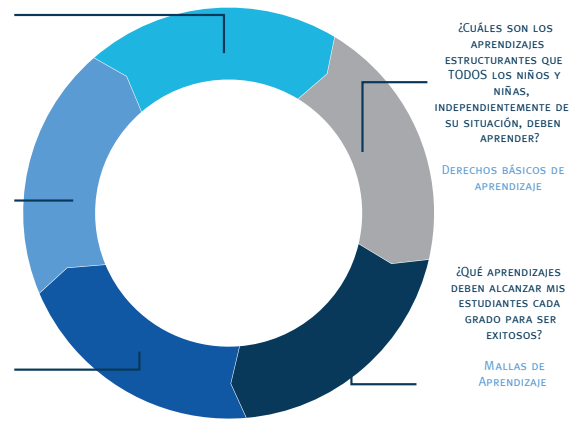

Fuente: Programa para la transformación de la calidad educativa, "Todos a Aprender" (2017).

Queda claro, entonces, que la formación de docentes de matemáticas y lenguaje es un tópico de las políticas educativas nacionales orientadas a la consolidación de un sistema que propenda por la calidad de la educación y, por ende, a mejorar los resultados en las pruebas estandarizadas. Ante esto, es importante resaltar que los procesos de formación de docentes de matemáticas y lenguaje en Colombia se dan en tres niveles: Educación Básica Secundaria (grados $6^{\circ}$ a $9^{\circ}$ ), Media (grados $10^{\circ} \mathrm{y}$ $\left.11^{\circ}\right), y$ Educación Básica Primaria (grados $1^{\circ}$ a $\left.5^{\circ}\right)(\mathrm{Gua}-$ caneme, Bautista y Salazar, 2011).

De lo anterior se puede deducir que existen divisiones marcadas en torno a los procesos de formación, lo cual genera problemas en tres grandes bloques: el primero, el relacionado con las dificultades epistemológicas que tienen los futuros docentes, lo cual se vincula con las creencias y concepciones (Da Ponte,1999), o en palabras de René Thom (1973), citado por Da Ponte (1999): “(...) detrás de cualquier modelo de enseñanza de las matemáticas hay una filosofía de la matemática (...)” (p.1). En segundo lugar, con el equilibrio entre las creencias y concepciones de los docentes y su relación con las prácticas (Bishaw, 2011; Pajares, 1992) y, por último, cómo estas prácticas inciden en el proceso de enseñanza y aprendizaje (Donoso, Rico y Castro, 2016), y por consiguiente en los resultados de las evaluaciones, tanto externas como internas. Ante este último aspecto, es importante destacar cómo Donoso, Rico y Castro (2016, pág. 78) muestran que existen “investigaciones (Aguilar, 2003; Benítez, 2013; Contreras, 2009; Donoso, Rico y Castro, 2016; Gamboa, 2014; Gil, 1999; Gil y Rico, 2003; Moreno y Azcárate, 2003; Thomaz, 
Cruz, Martins y Cachapuz, 1996)", que resaltan cómo las creencias y concepciones de los docentes inciden de forma directa en todos los procesos de enseñanza aprendizaje, sin dejar por fuera el rendimiento académico de los estudiantes.

\section{CREENCLAS, CONCEPCIONES Y LAS COMPETENCLAS DE FORMACIÓN DOCENTE.}

A continuación, se mencionan los principales hallazgos en torno a estos tres grandes bloques, y las diferentes estrategias que se han venido desarrollando por parte de las comunidades académicas para resolver dichos problemas. En primera medida, es significativo mostrar las posturas teóricas relacionadas con la formación docente y sus aproximaciones epistemológicas, entre ellas vale destacar las perspectivas teóricas basadas en contextos sociológicos y antropológicos (Malagón Patiño, 2013), como lo es la Teoría Antropológica de lo Didáctico (TAD) (Chevallard, 1999, 2001, 2005), Chevallard (1999), en la cual se muestra la actividad de enseñar y formar "maestros", no como una "actividad individual" sino como un "sistema de Prácticas" (Chevallard, 1999, p.223), desarrolladas por los docentes en una institución social, denominada "escuela". Ante esto, Malagón Patiño (2013) destaca cómo "la enseñanza no depende de los sujetos individualmente" (Chevallard, 1999, p.225), sino que parte de una interacción: maestros y su experiencia al ser formados y maestros al relacionarse con otros maestros. Ahora bien, en el caso de los docentes de matemáticas, esta "práctica" se nutre de otros dos aspectos, según Da Ponte (2004): "el primero se basa en la recurrencia sistemática y la segunda en el papel de reconocimiento y diferenciado" (p.4), que se establece dentro de la sociedad y de la institución escuela como tal.

En consecuencia con lo dicho, y atendiendo el eje de interacción relacionado con la experiencia al ser formados, es necesario destacar cómo la estructura de los procesos de formación de maestros de matemáticas en Colombia está organizada desde cuatro elementos fundamentales: La educabilidad, la enseñabilidad, la estructura histórica y epistemológica de la pedagogía, y las realidades sociales y educativas, dados estos por el decreto 272 de 1998 en su artículo $4^{\circ}$. Ahora bien, en el texto hecho por Guacaneme, Bautista y Salazar (2011), titulado "Informe sobre la formación inicial y continua de profesores de matemáticas: el caso de Colombia”, se logró determinar que las estructuras de los programas de formación de maestros en matemáticas giran en torno a líneas curriculares relacionadas con: “(...) la formación Matemática, el conocimiento curricular y el conocimiento Didáctico de las Matemáticas, el conocimiento pedagógico general y una línea centrada en aspectos comunicativos (...)" (Guacaneme et al., 2011, p.34).

Con respecto a lo anterior, es relevante decir que, a nivel nacional, se plantea un cambio de paradigma que coadyuve a mejorar estos procesos, y por tal razón se inicie la implementación de políticas para el desarrollo de competencias, porque según Tobón, Pimienta y García (2010), las competencias permiten o brindan "respuestas pertinentes y claras en torno al currículo, el aprendizaje, la evaluación y la gestión educativa-docente" (p.3). Así mismo, permiten afrontar problemas relacionados con "la relación entre currículo y microcurrículo, para asegurar la calidad educativa o la formación en torno a resolver problemas, donde se involucra el saber ser, el saber hacer y el saber conocer" (Tobón, Pimienta y García, 2010, p.4). Para esto, se empieza con la elaboración de documentos "maestros" que dinamizan los procesos de enseñanza y aprendizaje, y en primera instancia surgen los lineamientos curriculares como una "guía" estructurada que busca:

(...) incrementar la formación de quienes hacen currículo y de quienes asesoran a las instituciones educativas para que lleven a cabo sus procesos curriculares dentro del Proyecto Educativo Institucional. Deben servir de orientación, pero no reemplazan a los docentes en las decisiones que les corresponde tomar en asuntos como contenidos, metodologías y estrategias para la participación (...)

(Documento Lineamientos Curriculares, 1998, p.7)

De esto se desprende que los lineamientos en palabras de Rico (1995), sirven para organizar los procesos de ejecución de competencias, y en matemáticas se denomina lograr la "ejecución de tareas matemáticas" o los procedimientos o "modos de saber hacer" (Rico, 1995). Es así que los lineamientos se convierten en la base para la construcción de una definición más amplia y profunda de Competencia, surgiendo así el documento "Estándares Básicos de Competencia” (MEN, 2006), donde define las competencias:

“(...) como un conjunto de conocimientos, habilidades, actitudes, comprensiones $\mathrm{y}$
I Panorama

I pp.7-17

I Volumen 12

I Número 22

I Enero-Junio I 2018 
Alfredo González, Alex Díaz I

Panorama I

pp. 7-17

Volumen 12

Número 22 I

Enero-Junio

ISSN Impreso

1909-7433

E-ISSN en linea

2145-308X

$14 \mid$ disposiciones cognitivas, socioafectivas $y$ psicomotoras apropiadamente relacionadas entre sí para facilitar el desempeño flexible, eficaz y con sentido de una actividad en contextos relativamente nuevos y retadores (...)"(E.B.C, 2006, p.49).

Por último, es importante mencionar que la implementación de las políticas de competencias choca, en primera medida, con las creencias y concepciones de los maestros; al respecto, Thompson (1992), citado por González (2015), en su mayoría consideran las matemáticas como un cuerpo "estático del conocimiento, en el cual se aplican una serie de normas y procedimientos para emitir una apropiada respuesta" (p.13). Debido a estas concepciones, es clave destacar que dichas políticas no son suficientes para transformar su praxis como tal. A continuación, se amplía la información sobre este aspecto.

\section{CREENCIAS, CONCEPCIONES Y SU RELACIÓN CON LAS PRÁCTICAS Y SU INCIDENCIA EN EL PROCESO DE ENSEÑANZA-APRENDIZAJE}

Como se nota, la política educativa intenta modificar los procesos de enseñanza-aprendizaje, aunque se encuentra con un obstáculo relacionado con las creencias y las concepciones de los docentes, como lo plantea González (2015), al citar a Barrantes y Blanco (2004), que afirman: “(...) los docentes no desarrollan su trabajo mecánicamente, sino que bajo de sus acciones subyacen creencias que influyen sobre la enseñanza (...)" (González, 2015, p. 5). Al respecto, se plantean estrategias o propuestas didácticas que apuntan a la resolución de problemas, como pilar principal, donde se pretende generar procesos de reflexión del docente sobre su praxis, en palabras de Ramos, Flórez, Da Ponte y Moreno (2015): “(...) los cambios en la actuación de los docentes pueden verse reflejados en relación con las tareas para el aula diseñadas por ellos para afrontar su práctica (...)" (p.390).

Al respecto, se tiene que destacar que esta estrategia de resolución de problemas se articula con la definición dada por el MEN (2006), en el marco del documento de competencias y apunta a la construcción del conocimiento matemático. Aquí se destacan dos aspectos: el primero que apunta a los conocimientos conceptuales, “(...) el cual es teórico, producido por la actividad cognitiva, muy rico en relaciones entre sus componentes y con otros conocimientos; tiene un carácter declarativo y se asocia con el saber qué y el saber por qué" (MEN, E.B.C, 2006, p.50). En este sentido, se busca que se rompa el modelo tradicional, el cual no genera reflexión, porque la educación parte de "un docente que selecciona los contenidos de un programa, los organiza según su punto de vista y los explica” (Tobón, Pimienta y García, 2010, p.5), y se pase a un modelo donde puedan -tanto el estudiante, como el docente- "enfrentarse a una tarea relevante (situada) que generará aprendizaje por la "puesta en marcha” de todo el "ser"' (Pimienta y Enríquez, 2009, p.27).

Siguiendo a Pimienta y Enríquez (2009), en lo relacionado con el enfrentarse a una tarea relevante (situada), existen investigaciones que demuestran que estos profesores apuntan sus prácticas a procesos de reflexión e investigación, “logrando así una visión más dinámica de las matemáticas, logrando la conceptualización en sus estudiantes" (Prawat, 1992, citado por González, 2015, p.15), y observan las matemáticas como una herramienta para "resolver problemas de entendimientos culturales" (Thompson, 1992, citado por González, 2015, p.17). Debido a esto, se recomienda que las prácticas de aula se enfoquen a la construcción de conceptos matemáticos, al igual que actividades que generen razonamiento y de creatividad, a la recolección de información el descubrimiento y la comunicación de ideas; al respecto, González (2015) cita a Ball (1993); Cobb, Madera, Yackel, y Mcneal, (1993); Thompson (1993); Fenema, Carpenter, Franke y Carey (1993); Lambert (1991); Wood, Cobb y Yackel (1991).

En torno a este aspecto es importante mostrar que a nivel mundial se han diseñado programas que apuntan a la transformación de las prácticas de los docentes, como en el caso del programa Todos a Aprender, que busca: “(...) mejorar los resultados de los estudiantes a través del mejoramiento de las prácticas de aula" (CME 2025, p.20). Sobre esto, las investigaciones muestran que estos programas, según Guskey, citado por Malagón Patiño (2013): “(...) por sí mismos no generan cambios en las actitudes y creencias de los maestros, se requiere de experiencias de implementación exitosas para poder lograr estos cambios y esto se da cuando hay evidencias de una clara mejoría en los resultados de los aprendizajes de los estudiantes" (p.4).

De este modo, el rol del docente se entiende como un proceso largo y constante que trasciende hasta cualquier 
contexto que esté asociado con el proceso de enseñanzaaprendizaje (Johnson y Golombek, 2011). Sin embargo, los estudios abordados en esta revisión bibliográfica demuestran cómo el DPD se ejecuta en realidades educativas con distintas necesidades, obteniendo positivos resultados en el crecimiento profesional, académico y cultural de los docentes que hacen parte de estos tipos de entrenamiento. Por tanto, es pertinente proponer estas estrategias de desarrollo profesional docente, dado que permiten dar cuenta de la relación que existe entre el aprendizaje cooperativo y colaborativo de los pares docentes para el mejoramiento de los procesos de comprensión de lectura y de géneros textuales.

Figura 2. Sistema de relaciones esenciales para el Desarrollo Profesional Docente (DPD).

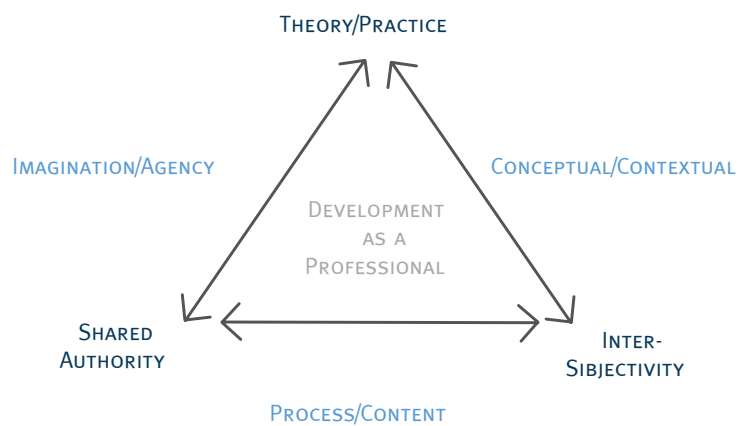

Fuente: Grimmet, (2014). The Practice of teachers' professional development.

Para resumir, en esta figura el desarrollo profesional docente está ubicado en el centro, como resultado de la práctica institucional, dado que no solo se exige que los docentes y las instituciones se comprometan con este proceso, también debe ser percibido por los docentes como un proceso relevante para su contexto, y como producto el mejoramiento significativo de la enseñanza en el contexto local (Yamagata-Lynch y Haudenschild, 2006). La mayoría de las prácticas curriculares de docentes y escuelas parecen estar orientadas a mantener un entorno de clase organizado y contextualizado, que al mismo tiempo cubre el currículo requerido para aprovechar la curiosidad natural y la sed de aprendizaje de los estudiantes (Grimmett, 2014). Igualmente, Grimmett argumenta que los profesores capaces de mantener la curiosidad y la motivación del alumno tienen más probabilidades de conservar su propia pasión por la enseñanza, en lugar de marchitarse en una vida profesional aburrida.

El DPD, de acuerdo con Grimmett (2014) es reconocido como un proceso complejo y arduo que no tiene punto de inicio o final. El anterior diagrama evidencia que el proceso DPD inicia la triangulación a partir de que los docentes se encuentran en su proceso de formación inicial e ingresan al aula por primera vez, hasta en las situaciones donde un maestro experimentado intenta comprender y dar solución a un problema en el aula, lo cual está catalogado como proceso sistémico de intersubjetividad y autoridad compartida. Además, en este tipo de investigaciones relacionadas con el DPD se ha descubierto que algunas escuelas están avanzando hacia iniciativas que brindan una visión más didáctica de la enseñanza y del aprendizaje, en la que los maestros pueden participar en un papel más activo en relación con su propio desarrollo profesional, trabajando junto a otros en su profesión para abordar varios problemas pedagógicos desde comunidades de aprendizaje (Clark, 2001).

Para finalizar, luego de esta revisión de literatura se pueden expresar algunas apreciaciones con respecto a cuáles son los cambios que se dan en la práctica pedagógica de los docentes luego de su formación en la enseñanza del lenguaje y matemáticas, tomando como documentos orientados de la acción pedagógica los referentes curriculares del sistema educativo colombiano. Por ejemplo, es factible afirmar que la gran mayoría de estudios demuestran la preocupación y el interés de todos los actores del sistema educativo por lograr el mejoramiento de la instrucción de la enseñanza y del aprendizaje de competencias lectoras y matemáticas, necesarias para el mejoramiento de la calidad educativa a nivel regional, nacional e internacional, desde la educación inicial en las áreas de lenguaje y matemáticas. De este modo, las prácticas educativas están definidas por currículo, didáctica y conciencia de oficio. Estas concepciones usualmente conllevan ideas, principios y acciones que todos los docentes deben practicar constantemente en las instituciones educativas. Por esta razón, se puede decir que las políticas educativas de formación docente y práctica pedagógica para la enseñanza de lenguaje y matemáticas están orientadas desde el Ministerio de Educación Nacional y las facultades de educación de las universidades del país hacia el aprendizaje cooperativo, colaborativo, desarrollo profesional situado y pedagógico de la siguiente manera:

A. a. Desarrollo de procesos participativos que desembocan en propuestas prácticas, mediante métodos cualitativos donde la participación de la comunidad de aprendizaje permite el desarrollo de procesos académicos y culturales como base para
Formación

docente y

desarrollo

profesional

situado para

la enseñanza del lenguaje y matemáticas en Colombia
I Panorama

I pp.7-17

I Volumen 12

I Número 22

I Enero-Junio

| 2018

15 
Panorama I

pp. 7-17

Volumen 12

Número 221

Enero-Junio

2018 |

ISSN Impreso

1909-7433

E-ISSN en linea

$2145-308 \times 1$

6. Chevallard,Y. (1999). El análisis de las prácticas docentes en la teoría antropológica de lo didáctico. Recherches en Didactique des Mathématiques, 19(2), 221-266.

3. Beachum, F., y Dentith, A. (2004). Teacher 276-286. Taylor \& Francis Group.

4. Cadavid, L. (2013). Del trabajo en equipo al Especial.p.146-159. Colombia - Agencia JICA
7. Da Ponte, J. P. (1999). Las creencias y concepciones de Maestros como un tema fundamental en formación de maestros. En Krainer y Goofree (1999) (Eds), On research in teacher education: From a study of teaching practices to issues in teacher education (p. 43-50).

8. Díaz, A. (2015). Evaluación del impacto del programa Todos a aprender en los procesos de enseñanza del lenguaje de las matemáticas. El caso de la Institución Educativa San José del pantano", Panorama (9), 17.

9. Donoso, P., Rico, N. y Castro, E. (2016). Ceencias y concepciones de Profesores Chilenos sobre las Matemáticas. Profesorado. Revista de Curriculum y Formación de Profesorado, 20(2), 76-97.

10. Furman, M. (2013). Programa de Educación Rural-PER: Orientaciones técnicas para la producción de secuencias didácticas para un desarrollo profesional situado en las áreas de Matemáticas y Ciencias. Bogotá: Ministerio de Educación Nacional de Colombia. implementación de procesos de investioación que van de la mano de las comunidades de aprendizaje, que se basan en la solución de problemas como enfoque de enseñanza y aprendizaje.

E. e. La implementación de procesos de sistematización de las prácticas de los docentes, tanto de matemáticas como de lenguaje, que permiten reflexiones sobre el diseño de estrategias de aula para mejorar los resultados y para la construcción de conceptos y el desarrollo de competencias.

\section{REFERENCIAS}

1. Abell, S., Rogers, M., Hanuscin, D. y Gagnon, M. (2009). Preparing the next generation of science teacher educators: a model for developing pack for teaching science teachers. Journal of Science Teacher Education, p.20.

2. Allen, D. y Blythe, T. (2004). The Facilitator's Book of Questions: Resources for Looking Together at Student and Teacher Work. Teachers College Press. leaders creating cultures of school renewal and transformation. The Educational Forum, 68(3), trabajo colaborativo. Revista Aletheia. Edición

5. Calvache y Escobar. (2007). Experiencia No. 5: 'Estudio de clase - Transformaciones Rígidas' Cauca - Año 2007. Nivel Regional. Convenio MEN
11. Glatthorn, A. (1987). Cooperative professional development: Peer-centered options for teacher growth. Educational leadership, 45(3), 31-35.

12. González,M. (2015). Creencias y Concepciones de los profesores sobre las matemáticas y su enseñanza-aprendizaje. Tesis de Maestría, Ciencias Sociales. Huelva: Univerisdad De Huelva.

13. Guacaneme, E., Obando, G., Garzón, D. y Villa-Ochoa, J.'(2013). Informe final sobre la formación inicial y continua de profesores de matemáticas: el caso de Colombia. Cuadernos de Formación e Investigación en Educación Matemática, 11-49.

14. Grimmett, H. (2014). The Practice of Teachers Professional Development: A Cultural-Historical Approach. Springer.

15. Grossman, P.; Hammerness, K. y McDonald, M. (2009). Redefining teaching, re-imagining teacher education. Teachers and teaching: theory and practice, 15(2), 273-289.

16. Johnson, K. y Golombek, P. (2011). Research on second language teacher education: A sociocultural perspective on professional development. New York: Routledge.

17. Keenan, C. (1974). Channels for Change, a Survey of Teachers in Chicago Elementary Schools. Urbana.

18. Malagón Patiño, M. (2013). Los programas de formación de maestros de matemáticas y su relación con las prácticas docentes. I Congreso de Educación Matemática de América Central y del Caribe. Santo Domingo, República Dominicana. 
19. Marchesi, A (2011). Preámbulo. En: Vélaz de Medrano, C. y Vaillant, C. Aprendizaje y desarrollo profesional docente. Madrid: Organización de Estados Iberoamericanos para la Educación, la Ciencia y la Cultura, OEI, p.7.

Formación
docente y
desarrollo

profesional

situado para

la enseñanza

del lenguaje y

matemáticas en

Colombia.

22. Ministerio de Educación Nacional, MEN. (2015). Colombia, la mejor educada en el 2025. líneas estratégicas de la politica educativa del Ministerio de Educación Nacional. Bogotá.

23. Pajares, F. (1992). Teacher's beliefs and educational reserarch: cleaning up a messy construct. Review of Educational Research, 62(3), 307-332.

24. Pimienta, J.y Enríquez, A. (2009). Educación basada en competencias. Guia para la aplicación del enfoque. México: Pearson.

25. Poehner, P. (2011). Teacher Learning through Critical Friends Groups. Research on second language teacher education: A sociocultural perspective on professional development, 189-203.

26. Putman, R. y Borko, H. (2000). What do new views of knowledge and thinking have to say about research on teacher learning? Educational Research, 29(1), 4-15.

27. Ramos, E., Flores, P. y Da Ponte, J. (2015). Desarrollo profesional del docente de matemáticas a través de sus tareas para el aula propuestas en un curso de formación. Bolema, 389-402.

28. Rico, L. (1995). Errores y dificultades en el aprendizaje de las matemáticas. Bogotá: Universidad de los Andes.

29. Shulman, L. (2005). Conocimiento y enseñanza: fundamentos de la nueva reforma. Revista de Curriculo y Formación del Profesorado, 9(2).

30. Tobón, S., Pimienta, J. y García, J. (2010). Bases teóricas y filosóficas de la formación de las competencias. Secuencias Didácticas: aprendizaje y evaluación de competencias, 44-48.

31. UNESCO (2014). Enseñanza y Aprendizaje: Lograr la calidad para todos. París: Ediciones UNESCO.

32. Yamagata-Lynch, L. y Haudenschild, M. (2006). 